Check for updates

Cite this: RSC Adv., 2018, 8, 40778

Received 4th October 2018

Accepted 24th November 2018

DOI: $10.1039 / c 8 r a 08218 a$

rsc.li/rsc-advances

\section{NMR insights on nano silver post-surgical treatment of superficial caseous lymphadenitis in small ruminants $\dagger$}

Danijela Stanisic, (1D *a Natália L. Fregonesi, ${ }^{\text {a }}$ Caio H. N. Barros, ${ }^{a}$ João G. M. Pontes, ${ }^{a}$ Stephanie Fulaz, ${ }^{a}$ Ulisses J. Menezes, ${ }^{\text {b }}$ Jorge L. Nicoleti, ${ }^{\text {b }}$ Thiago L. P. Castro, ${ }^{c}$ Núbia Seyffert, ${ }^{d}$ Vasco Azevedo, ${ }^{c}$ Nelson Durán, ${ }^{\text {ef }}$ Ricardo W. Portela ${ }^{b}$ and Ljubica Tasic (D) *ae

Caseous lymphadenitis (CL), caused by a pathogen of the second class of biosafety - Corynebacterium pseudotuberculosis, is a chronic and severe infectious disease that affects small ruminants and requires long, ineffective treatment which generally leads to animal sacrifice so as to stop the disease spreading. The infected animals suffer the excision of affected superficial lymph nodes and post-surgical treatment with iodine ( $10 \%$ solution in ethanol) and, sometimes, prolonged antibiotic use, but only if the sick animals are of great importance to breeding. Herein, we propose a cheap and easy to apply treatment of CL with excellent results using biogenic silver nanoparticles (AgNP) based technology. AgNP antibacterial properties were investigated in vitro against Corynebacterium pseudotuberculosis cells and in vivo on small ruminants with $\mathrm{CL}$. Treatment of surgical wounds resulting from the excision of superficial $\mathrm{CL}$ lesions with a AgNP-based cream was compared to the standard post-surgical treatment method by iodine. Also, the effects of AgNP-based cream treatment were evaluated and compared with the effects of the iodine $C L$ treatment by serum NMR-based metabolomics. Serum samples were collected from 29 animals, 9 sheep and 20 goats, during the treatments and analyzed. All animals showed stable serum metabolomes when iodine or AgNP-based cream effects were compared. The AgNP-based cream treatment showed excellent results, especially in accelerating the healing of wounds, which occurred two to three times faster in comparison with the iodine treatment. AgNPbased cream treatment also prevented $\mathrm{CL}$ reappearance and did not cause any side effects on animals. This is the first report on very effective post-surgical treatment of superficial $C L$ in small ruminants based on biogenic silver nanoparticles, which might open up the possibility for a safe veterinary application of AgNP-based cream.

\section{Introduction}

Corynebacterium pseudotuberculosis (C. pseudotuberculosis) is a Gram positive, ${ }^{\mathbf{1} 2}$ non-encapsulated, facultative and intracellular bacterium, which is the etiological agent of caseous

${ }^{a}$ Laboratório de Química Biológica, Departamento de Química Orgânica, Instituto de Quimica, Universidade Estadual de Campinas, Campinas, SP, Brazil. E-mail: ljubica@iqm.unicamp.br

${ }^{b}$ Laboratório de Imunologia e Biologia Molecular, Instituto de Ciências da Saúde, Universidade Federal da Bahia, Salvador, BA, Brazil

'Departamento de Biologia Geral, Instituto de Ciências Biológicas, Universidade Federal de Minas Gerais, Belo Horizonte, MG, Brazil

${ }^{d}$ Laboratório de Bacteriologia e Saúde, Instituto de Biologia, Universidade Federal da Bahia, Brazil

${ }^{e}$ NanoBioss - Institute of Chemistry, University of Campinas, Campinas, SP, Brazil ${ }^{f} U F A B C$, São Paulo, SP, Brazil

$\dagger$ Electronic supplementary information (ESI) available. See DOI: 10.1039/c8ra08218a lymphadenitis (CL) in small ruminants (biovar ovis) and ulcerative lymphadenitis in equines (biovar equi). ${ }^{3}$ This disease provokes great economic losses worldwide and affects wool, meat and milk production., ${ }^{3,4}$ Many efforts have been made toward a better understanding of the C. pseudotuberculosis pathogenicity through genomics, proteomics and metabolomics, ${ }^{1,5,6}$ but the host-microorganism interaction mechanisms in CL are still not understood. Also, C. pseudotuberculosis is not an easy target for antibiotics. The encapsulated lesions in CL do not allow an easy penetration of drugs, thus, treatment is long and not effective in most cases. There are many efforts towards finding better CL treatments, for example, in vitro evaluation of antimicrobial susceptibility of C. pseudotuberculosis using a variety of antibiotics. ${ }^{7,8}$ Recently, silver and gold nanoparticles were tested ${ }^{9-11}$ and their antimicrobial activities against the C. pseudotuberculosis $^{11}$ were reported. The current treatment of the CL depends on excision of lesions in superficial lymph nodes and the use 
of an iodine solution ( $10 \%$ in $70 \%$ of ethanol) for sterilization of surgical wounds, but iodine showed to be histotoxic and prolonged the wound healing process. ${ }^{\mathbf{1 2}}$

Silver antimicrobial activity is antique ${ }^{\mathbf{1 0}}$ and silver(I) has been used for treatment of wounds and ulcers from $18^{\text {th }}$ century. ${ }^{13}$ The bactericidal effects of silver(I) had been described for many microorganisms, such as Escherichia coli ${ }^{\mathbf{1 4}}$ Staphylococcus aureus, ${ }^{\mathbf{1 5 , 1 6}}$ Candia albicans, Xanthomonas axonopodis pv. citri $^{\mathbf{1 7}}$ and others. ${ }^{\mathbf{1 8 - 2 0}}$ However, the action mechanism of silver(I) is not fully understood. ${ }^{21}$ In addition, silver nanoparticles (AgNPs) ${ }^{22-27}$ have a pronounced antibiotic activity and can decrease the inflammatory cascade and tissue proliferation. The last two processes count on fibroblast cells activity that leads to a faster wound healing and formation of more organized collagen fibres without creating scars. $^{23}$

Therefore, we hypothesized that AgNP application might assist in CL treatment in a practical and a cheap way if biobased particles could be applied, as to avoid the use of chemically synthesized AgNPs, which might have undesired effects, even in regard of environmental issues. But before recommended for use for animal and/or human treatment, the AgNP toxicity needs to be tested.

The current study aimed to formulate the biogenic AgNPbased skin-friendly cream for dermal use and apply it on post-surgery wounds of CL-suffering animals. Also, the effectiveness of AgNP-based cream was compared with the CL iodine treatment. Eventual toxic effects of AgNPs on treated animals were monitored through biochemical tests and serum metabolomics by NMR. ${ }^{6,28-37}$ Two types of AgNPs were tested, biogenic Fusarium oxysporum ones and bio-based AgNPs from Citrus sinensis peel extracts. ${ }^{\mathbf{1 8 , 3 8}}$

\section{Materials and methods}

\section{Biogenic silver nanoparticles (AgNP) syntheses}

The synthesis of AgNPs from Fusarium oxysporum was done based on the procedure described by Ballottin et al. (2017). ${ }^{17}$ Briefly, $10 \mathrm{~g}$ of fungal filtrate (Fusarium oxysporum) was mixed with a silver nitrate solution $\left(1 \mathrm{mmol} \mathrm{L}^{-1}\right)$, which formed nano silver in $72 \mathrm{~h}$ in the dark. ${ }^{17}$

On the other hand, the synthesis of AgNPs with orange peel extract was performed as described by Barros et al. (2018). ${ }^{38}$ Briefly, an orange peel extract was made by mixing $50 \mathrm{~g}$ of chopped peel with water and after heating it up to the boiling point. Then, using filtration and a $0.22 \mu \mathrm{m}$ pore membrane, the orange peel extract was obtained and added to a silver nitrate solution $\left(1 \mathrm{mmol} \mathrm{L}^{-1}\right)$ and kept in the dark for $48 \mathrm{~h}^{38}$

These nano silver particles ${ }^{17,38}$ were characterized and showed the following physical-chemical properties: (a) F. oxysporum AgNPs had sizes of $28.0 \pm 13.1 \mathrm{~nm}$, showed polydispersity of 0.231 , zeta potential of $-31.7 \pm 2.8 \mathrm{mV}$, and were spherical in form; (b) orange peel AgNPs had sizes of $48.1 \pm$ $20.5 \mathrm{~nm}$ and polydispersity of 0.312 , zeta potential of $-19.0 \pm$ $0.4 \mathrm{mV}$, also, were spherical.

\section{C. pseudotuberculosis cells and AgNP activities}

The C. pseudotuberculosis strain 1002 was provided by Laboratório de Genética Celular e Molecular (LGCM, UFMG, Belo Horizonte, Brazil) and maintained in glycerol at $-80{ }^{\circ} \mathrm{C}$, until cultivation in Brain Heart Infusion (BHI) broth at $37^{\circ} \mathrm{C}$ for $40 \mathrm{~h}$. C. pseudotuberculosis was grown in the culture medium, centrifugated and transferred to a $\mathrm{NaCl}$ solution (0.9\%) with dilution monitored by turbidity at $600 \mathrm{~nm}(0.1)$. Then, bacteria were pipetted in sterile 96-well plates.

A volume of $100 \mu \mathrm{L}$ bacterial culture and $100 \mu \mathrm{L}$ of sterile medium culture was pipetted in the first row of the plate (Positive Control) and in row 2, $100 \mu \mathrm{L}$ of AgNP suspension and $100 \mu \mathrm{L}$ of sterile culture medium were added (Negative Control). In row 3, $200 \mu \mathrm{L}$ of the AgNP $268 \mu \mathrm{g} \mathrm{mL}^{-1}$ were added and from rows 4 to 12, the concentrations of AgNPs were adjusted to 28, $26,24,22,20,18,16,14,12 \mu \mathrm{g} \mathrm{mL}^{-1}$ using sterile culture medium. In the case of orange extract based AgNPs, the stock suspension of $54 \mu \mathrm{g} \mathrm{mL}^{-1}$ was also diluted through rows 4 to 12 , reaching concentrations of $35,30,25,20,15,5,2$ and $1 \mu \mathrm{g} \mathrm{mL} L^{-1}$. $50 \mu \mathrm{L}$ of bacterial culture (turbidity at $600 \mathrm{~nm}$ was 0.1 ) were added to the wells of rows 4 through 12 . The plates were incubated at $37^{\circ} \mathrm{C}$ for $48 \mathrm{~h}$, and bacterial growth was monitored by visual detection of turbidity. ${ }^{39}$

\section{Scanning electron microscopy analyses}

Bacterial suspensions in conditions mimicking the MIC assays at the MIC and one half of MIC concentration were deposited in $0.45 \mu \mathrm{m}$ pore sized, $25 \mathrm{~mm}$ PTFE membrane (Millipore ${ }^{\circledR}$ ), fixed with $2.5 \%$ glutaraldehyde solution in sodium phosphate buffer at $\mathrm{pH} 7.2$ and washed twice with PBS $(\mathrm{pH}=7.4)$ buffer. The membrane was soaked with osmium tetroxide $1.0 \%$ for $1 \mathrm{~h}$ and washed twice with distilled water. The dehydration process consisted in immersing the membrane in ethanol $30 \%$ for $20 \mathrm{~min}, 50 \%$ for $20 \mathrm{~min}$; $70 \%$ for $16 \mathrm{~h}$; $90 \%$ for $20 \mathrm{~min}$ and three times in ethanol p.a. for $20 \mathrm{~min}$. The drying was performed in a Critical Point Dryer (CPD) (Balzers ${ }^{\circledR}$ CPD-0.30), the sputtering with gold by Sputter Coater SCD-050 and SEM analyses in a Jeol® JSM 5800LV microscope.

\section{AgNP-based cream formulation}

The cream formulations with AgNPs were made with a natural oily base and nanoparticles obtained by two described methods, which were incorporated after preparing the base. The cream in the water-in-oil base was prepared by heating the natural

Table 1 Composition of AgNP-based cream

\begin{tabular}{lllrl}
\hline Phase & Trade name & INCI name & w/w\% & Supplier \\
\hline A & Lanolin & Lanolin & 20.0 & Fagron \\
& Cera alba & Cera alba & 9.0 & Fagron \\
& Vaselinum solidum & Petroleum & 31.0 & Fagron \\
& Vaselinum liquidum & Paraffinum & 10.0 & Fagron \\
& & liquidum & & \\
& Cetostearyl alcohol & Cetostearyl alcohol & 8.0 & Fagron \\
& Cholesterol & Cholesterol & 2.0 & Fagron \\
B & AgNPs & AgNPs & 20.0 & Unicamp
\end{tabular}


ingredients (Table 1, formulation described in patent BR102017014836022) at $60{ }^{\circ} \mathrm{C}$ under stirring, followed by the addition of the AgNP colloid solutions at room temperature, adjusted to the MIC concentration.

A grey coloured cream with oily consistency, easy to apply and with the property to leave a greasy film on the skin was obtained.

\section{Nuclear magnetic resonance spectroscopy analyses}

Acquisition of the all 1D and 2D $\left({ }^{1} \mathrm{H}\right.$ and $\left.{ }^{13} \mathrm{C}\right)$ Nuclear Magnetic Resonance - NMR spectra were done on a Bruker Avance III 600 MHz spectrometer, utilizing TBI - Triple Resonance Broadband Inverse probe at $25^{\circ} \mathrm{C}$.

\section{NMR sample preparation for C. pseudotuberculosis analysis - in vitro}

For this NMR analysis and subsequent metabolomics, 3 distinct groups were studied. In all groups, C. pseudotuberculosis was grown in $10 \mathrm{~mL}$ of $\mathrm{BHI}$ media. For group 1, the bacterial cultures were grown for $24 \mathrm{~h}$ at $200 \mathrm{rpm}$ and $32{ }^{\circ} \mathrm{C}$. To the culture media of groups 2 and $3,10 \mathrm{~mL}$ of ampicillin $(0.25 \mu \mathrm{g}$ $\left.\mathrm{mL}^{-1}\right)$ and $10 \mathrm{~mL}$ of AgNPs $\left(20 \mu \mathrm{g} \mathrm{mL}^{-1}\right)$ were added, respectively, after $16 \mathrm{~h}$ of agitation. Then, the cultures were grown for another $8 \mathrm{~h}$, reaching turbidity at $600 \mathrm{~nm}$ of 0.8 .

By adding ethanol, in the ratio $1: 1(\mathrm{v} / \mathrm{v})$ to the bacterial cultures, the growth of $C$. pseudotuberculosis was quenched. Then, the suspensions were centrifuged for $10 \mathrm{~min}$ at $7000 \times g$ at $4{ }^{\circ} \mathrm{C}$, and the cells were suspended in $30 \mathrm{~mL}$ of ice-cold PBS $\left(20 \mathrm{mmol} \mathrm{L}^{-1}, \mathrm{pH}=7.2,0{ }^{\circ} \mathrm{C}\right)$; this step was repeated twice. The cells were lysed by ultrasonication for $10 \mathrm{~s}$ (Ultrasonique). The obtained biological material was centrifuged at $17000 \times g$ during $2 \mathrm{~min}$, then the supernatant was kept, cell residues were washed, and the supernatants were combined and lyophilized.

Samples for NMR were prepared by dissolving $15 \mathrm{mg}$ of the biological material into $500 \mu \mathrm{L}$ of deuterium oxide and spectra were acquired on a Bruker Avance III $600 \mathrm{MHz}$ spectrometer with TBI - probe at $25{ }^{\circ} \mathrm{C}$. ${ }^{1} \mathrm{H}$ NMR spectra were acquired as described in analysis section.

\section{Postsurgical wound treatment: $10 \%$ iodine solution and AgNP - based cream - in vivo assays}

Postsurgical treatment of caseous lymphadenitis in infected animals was conducted with 29 animals, 9 sheep and 20 goats in a research coordinated by Federal University of Bahia. All animals presented clinical symptoms of the disease and the presence of the bacteria in the affected lymph nodes was confirmed by microbiological assays. The animals underwent a surgical procedure that started with the trichotomy of the skin above the enlarged lymph node and disinfection with $70 \%$ ethanol. An incision was made with a sterile blade and all the caseous material was removed and collected in sterile vials for further microbiological processing and bacterial identification. The surgical wound was then treated with a $10 \%$ iodine solution in $70 \%$ ethanol (three times per week, for 8-10 weeks) or with the AgNP-based cream, one time just after the surgical treatment, the wound was filled with a cream to cover all the lesion. Veterinarians (Federal University of Bahia, UFBA, Salvador, BA, Brazil) monitored animals for 10 weeks after the treatment, and clinical parameters (body temperature, respiratory and cardiac frequency, hydration profile, colour of mucosa) were weekly taken and the size and profile of the wounds were recorded. This work was approved by the Committee on Ethics in Animal Experimentation of the Veterinary School of the Federal University of Bahia (number 35/2017).

\section{NMR analyses}

Blood samples were collected weekly from 29 animals per 10 weeks. Overall, we used sheep (5/9) that underwent AgNPbased cream treatment and $4 / 9$ that were treated with iodine. Goats (10/20) that underwent AgNP-based cream treatment and the other ten animals were treated with iodine. Serum samples were obtained by centrifugation and stored in a bio freezer at $-80{ }^{\circ} \mathrm{C}$ until the acquisition of NMR spectra. NMR analyses were done from serum prepared by dilution $1 / 1$ (v/v), with $250 \mu \mathrm{L}$ of deuterium oxide $\left(\mathrm{D}_{2} \mathrm{O} 99.9 \%\right.$, Sigma Aldrich) using $5 \mathrm{~mm}$ NMR tubes. ${ }^{1} \mathrm{H}$ NMR spectra were acquired using WATERGATE pulse sequence (p3919gp) with 128 scans, receptor gain (RG) of 101, FID size of 32768 in a spectral width of $12.02 \mathrm{ppm}$. The $\mathrm{CH}_{3}$ - lactate signal $(\mathrm{d}, 3 \mathrm{H}$, $\delta 1.33,{ }^{3} \mathrm{~J}=7.0 \mathrm{~Hz}$ ) was used as a reference. The $\mathrm{T}_{2}$-edited ${ }^{1} \mathrm{H}$ NMR spectra were acquired using the cpmg1d (Carr-Purcell Meiboom-Gill) pulse sequence with 256 scans, receptor gain (RG) of 203, FID size of 65536 in a spectral width of $20.55 \mathrm{ppm}$, 240 loops and processed with $\mathrm{LB}=1.00 \mathrm{~Hz}$. Two-dimensional spectra (HSQC) were acquired using the 600.17 MHz frequency domain spectrometer for F2 and $150.91 \mathrm{MHz}$ in the $\mathrm{F} 1$ domain with a free induction decay size (FID) of 4096 (F2) and 256 (F1) data points and 16 "ghost" scans were used. HSQC spectra were acquired with an acquisition time of $2.129 \times 10^{-1} \mathrm{~s}(\mathrm{~F} 2)$ and $4.437 \times 10^{-3} \mathrm{~s}$ (F1), using the hsqcedetgpsp.3 pulse sequence. The spectra were processed using $\mathrm{LB}=1.00$ for $\mathrm{F} 2$ and $\mathrm{LB}=0.30$ for $\mathrm{F} 1$. NMR acquired spectra were divided into two groups: sheep $(\mathrm{O})$ and goats $(\mathrm{C})$ group.

Both groups were classified by type of the treatment - iodine (10\%) (I) and AgNP-based cream (P), and all spectra were normalized by sum, with exclusion of residual water signal (HDO from $\delta 4.20$ to 5.50) and exported as matrix for chemometrics analyses using software MetaboAnalyst 3.0.

The samples from the $C$. pseudotuberculosis extract were divided into three groups. The first group refers to extract, the second group represents the extract of post-ampicillin treated bacteria and the third group - the extract with AgNP from orange peel.

\section{Inductively coupled plasma optical emission spectroscopy}

Presence of $\operatorname{Ag}(\mathrm{I})$ in animal serum samples was determined using Inductively Coupled Plasma Optical Emission Spectrometry (ICP-OES) technique, Perkin-Elmer - Optima 8300. An analytical curve, with silver(I) of ICP standard (Grupo Química, conc. $1000 \mathrm{mg} \mathrm{L}^{-1}, 1 \mathrm{~g} \mathrm{Ag}(\mathrm{I})$ per 2 to $5 \% \mathrm{HNO}_{3}$ ) was prepared 
and the serum sample from sheep 564 P_O, after application of AgNP-based cream was analysed.

\section{Results and discussion}

\section{Minimum inhibitory concentration and scanning electron microscopy}

The physical and chemical characterization of the nanoparticles used in this study can be found in previous articles. ${ }^{17,38}$ Inspection of C. pseudotuberculosis growth was determined visually in the plate wells. The rows in which the turbidity could not be observed (characteristic for bacterial growth) were considered as AgNP concentrations that completely inhibited the microorganism growth. The MIC value obtained for $C$. pseudotuberculosis of Fusarium oxysporum nanosilver was $33.5 \pm$ $2.0 \mu \mathrm{g} \mathrm{mL} \mathrm{m}^{-1}$, and of orange peel nanosilver was $22 \pm 2.0 \mu \mathrm{g}$ $\mathrm{mL}^{-1} .{ }^{22}$ In addition, AgNPs were bacteriostatic at the same concentrations.

The SEM images of C. pseudotuberculosis versus biogenic silver nanoparticles are presented in Fig. S1 (ESI†). It can be observed that there are bacterial clusters (spheres and rods) at AgNP MIC when compared to the other conditions. The cells appear to be deformed in an environment with organic matter, which may indicate the presence of cell debris. Some clear spots have been observed, which may be attributed to the nanoparticles, much smaller than the cells of $C$. pseudotuberculosis $(0.5-0.8 \mu \mathrm{m}$ per $1.0-3.0 \mu \mathrm{m})$. The mechanism of antimicrobial action of silver nanoparticles is not completely known, however, there are some theories that explain the performance of silver( $(\mathrm{I})$ and silver(0) on bacteria..$^{21,40-42}$

\section{Chemometrics on NMR data from test performed in vitro with C. pseudotuberculosis extracts}

Fig. 1 shows the superposition of ${ }^{1} \mathrm{H}$ NMR spectra obtained through in vitro tests with C. pseudotuberculosis, where AgNP effects were exposed in blue.

The PCA of ${ }^{1} \mathrm{H}$ NMR data from C. pseudotuberculosis extracts are shown in Fig. S2 (ESI†). The score graph points to a significant separation between the three groups. Group 1 (extract) separates well from the groups 2 and 3, in PC 1 . Groups 2 and 3

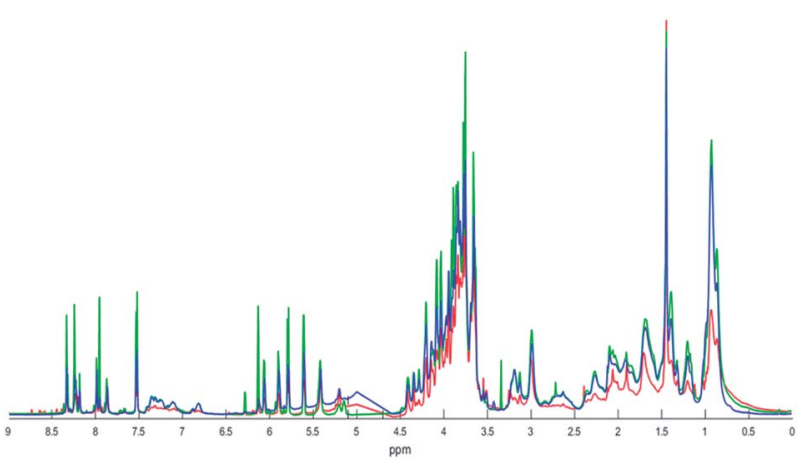

Fig. $1{ }^{1} \mathrm{H}$ NMR data of C. pseudotuberculosis extract (red line, 1), after addition of ampicillin (green line, 2) and after addition of AgNPs (blue line, 3). also show separation tendency but less pronounced, which is to be expected because they refer to the extracts treated with antimicrobials. The analysis of the loading graph (Fig. S2 $\dagger$ ) indicates that there are two regions responsible for the differentiation between the samples in PC 1. One of them varies from 0.91 to $0.94 \mathrm{ppm}$ and is in the positive region in PC 1 and at the $1.45 \mathrm{ppm}$ in negative PC 1 region. In PC 2 we also found the region of $1.45 \mathrm{ppm}$, but in this case, it showed positive loadings. Also, it was observed that chemical shift at $1.44 \mathrm{ppm}$ showed negative loadings. The metabolites with chemical shift at $1.45 \mathrm{ppm}$ were present in the highest concentration in the group 1 , whereas the metabolites in the region between 0.91 and $0.94 \mathrm{ppm}$ showed greater amounts in groups 2 and 3. It is important to note that these are relative concentrations because no quantitative experiments were per-formed. As PCA is only an exploratory analysis of the data, an analysis of PLS-DA was also performed.

The results of the PLS-DA model were similar to the PCA and there was also a significant separation between the groups, as can be observed in Fig. 2. Group 1 separates well from groups 2 and 3, in PC 1 (variance 29.8\%), while groups 2 and 3 were separated from each other in PC 2 (variance 19.6\%).

The analysis of the loading graph points to three important regions: (A) 0.91 and $0.94 \mathrm{ppm}$ in PC 1and (B) 1.44 and $1.45 \mathrm{ppm}$ in PC 1; and (C) 1.44 to $1.45 \mathrm{ppm}$ in PC 2. The chemical shifts around $0.90 \mathrm{ppm}$ refer to the lipid region and at $1.44 \mathrm{ppm}$ to aliphatic hydrogens from the carbon chains that belong to the mycolic acid, which is the main constituent of long chain lipids in cell wall of target mycobacteria..$^{4-45}$ The analysis of the VIP score graph (Fig. 3) confirms the conclusions obtained from the loading graph.

The importance of the variances with chemical shifts at 1.44 and $3.68 \mathrm{ppm}$ can be attributed to mycolic acid long chains from the cell wall in C. pseudotuberculosis extracts. Inosine and adenosine are also present in higher concentrations in extract treated with AgNPs, due to defence of bacteria against silver nanoparticles and higher production of extracellular adenosine. Mature products of adenosine synthases, in Gram-positive bacteria are localized in the bacterial cell-wall envelope and are responsible for the increase of extracellular concentrations of the potent immunosuppressive molecule adenosine and, through this mechanism, can perturb immune defences and
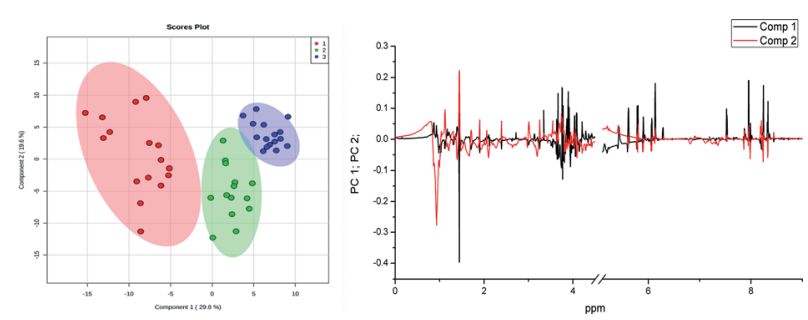

Fig. 2 Chemometrics on NMR data from tests with C. pseudotuberculosis: 2D scores plot of the PLS-DA analysis (left), with variance of $29.8 \%$ in PC 1 and $19.6 \%$ in PC 2, and the PLS-DA loadings graph (right). The red circles present the group of C. pseudotuberculosis extract, the green circles correspond to C. pseudotuberculosis extract after addition of ampicillin and blue circles represent the C. pseudotuberculosis after addition of AgNPs. 


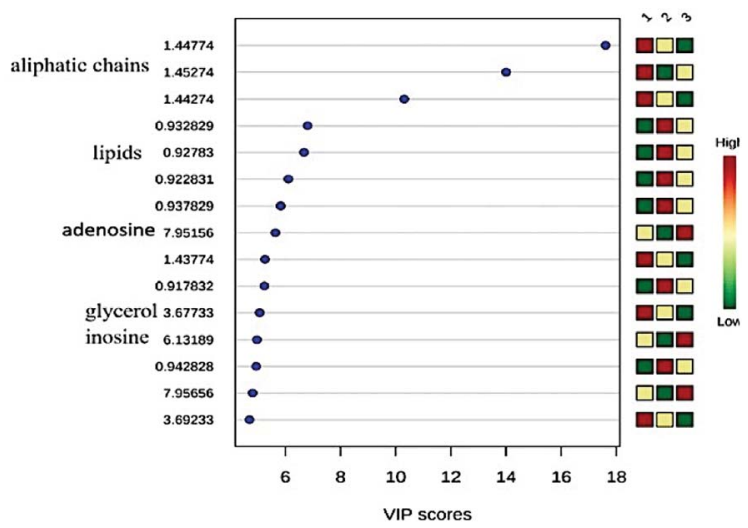

Fig. 3 Importance of the variables (VIP) obtained in PLS-DA model for the $C$. pseudotuberculosis extracts. The coloured boxes on the right indicate the relative concentrations of the corresponding metabolites in 1-3 groups. The numbers at the left side are chemical shifts of metabolites. The group 1 is C. pseudotuberculosis extract, group 2 correspond to C. pseudotuberculosis extract treated with the ampicillin and group 3 represents C. pseudotuberculosis extract after AgNP treatment.

promote their survival in host tissues. ${ }^{46} \mathrm{AgNPs}$ could induce cell death through increased membrane permeability and inactivation of the bacterial respiratory chain. ${ }^{47-49}$

The Fig. S3 (ESI†) represents the cross-validation of the PLSDA model. The value of $Q^{2}>0.4$ and the value of $R^{2}$ (significantly high) point to a valid model.

\section{Biogenic AgNP-based cream - effects on wounds}

A topical epidermal administration of water-in-oil cream emulsions or ointment as carriers of active substances enables their penetration to deeper layers of the animals' skin. Epidermal lipids are non-polar, composted from ceramides, sterol, fatty acids and lipophilic molecules and therefore, penetration through the stratum corneum is facilitated. ${ }^{50}$ After removal of nodules caused by the bacteria C. pseudotuberculosis, the animals were treated with AgNP-based cream or iodine solution and monitored for a period of ten weeks, in which iodine procedure was repeated three times per week. It has been observed that wounds were healed just after two weeks (Fig. 4) when AgNP-based cream treatment was used, differently for seen for iodine solution application that took a two to three times longer treatment period. Most of all, AgNP-based cream treated animals did not presented alterations of body temperature, respiratory and cardiac rate, mucosa coloration, as well as, there were no signals of photo-sensibilization in the treated skin and wound.

The action of the AgNP cream prevents the further formation of colonies of C. pseudotuberculosis as seen in MIC assays. In general, shallow skin lesions heal efficiently within the time of 2 weeks, compromising the epidermal cicatrix at the site of the injury. Most of the wounds do not fully regenerate and leave a connective tissue scar on the surface. Many wounds involve damage to blood vessels and provide a temporary repair, which initiates with hemostasis. ${ }^{26}$ In haemostasis, a fibrin clot temporarily plugs the damage site and, in subsequent days,

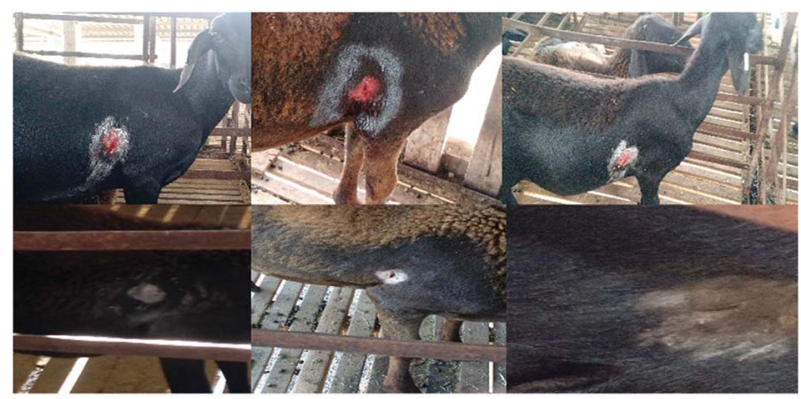

Fig. 4 Animals after chirurgical removal of nodules caused by the bacteria C. pseudotuberculosis (three sheep, first row). The same sheep are shown 2 weeks after application of AgNP-based cream (second row).

starts the healing process. There are five phases in wound healing: haemostasis, inflammation, cellular migration and proliferation, protein synthesis and wound contraction, and, lastly, remodelling. Because there is some overlap between these phases, they have been condensed into three phases: inflammation, proliferation, and remodelling. ${ }^{23,26}$ There is a reservoir of cytokines and growth factors released by activated degranulating platelets after clotting. During the inflammatory phase, these chemicals signal process and sequential infiltration of inflammatory cells (neutrophils, macrophages, and lymphocytes) at the wound site..$^{23,25,26,51}$ Neutrophils accumulate within minutes at the injury site to engulf and clear up any contaminating bacteria. Later, macrophage cells phagocytose neutrophils and apoptotic cells and undergo phenotypic transition to the reparative state that stimulates keratinocytes, fibroblasts and angiogenesis. Later, macrophage cells phagocytose neutrophils and apoptotic cells and undergo phenotypic transition to the reparative state, which stimulates keratinocytes, fibroblasts and angiogenesis. ${ }^{52}$ Herein, there are clear indications that silver incorporated into the oily base cream can promote wound healing, reduce the inflammatory phase and increase the proliferation time and re-modeling. ${ }^{23}$

It is very important to emphasize that the applied AgNPbased cream treatment had a positive effect on wound healing, which also has been shown in several papers. Recently, Stojkovska et al..$^{53}$ compared commercial treatment (Sulfadiazine cream 1\%) with wet and dry alginate microfibers with AgNPs of the second-degree thermal wounds in Wistar rats. Histopathological analyses pointed to improved granulation and reepithelization, organized extracellular matrices in a treated group, and no harmful effects of silver..$^{53}$ Similar, positive effects were demonstrated in experiment by Tian et $a l .{ }^{23}$ where treated burn wounds of Wistar rats with nanosilver dressings were monitored through cytokines' profiles during the treatment. Improved wound healing, reduced appearance of scars, decrease in inflammation-indicating cytokines (IL-10, IL6 , TGF- $\beta 1$, VEGF and IFN- $\gamma$ ), without any detrimental effect on the metabolism of the animals were reported. ${ }^{23}$ Moreover, there are results of various wound dressings, gaze, gels, solutions and creams based on nanosilver, which improve wound healing, increase rate of proliferation of the fibroblasts, accelerate 
epithelization and reveal a clean environment through the antimicrobial properties of silver(I) present. ${ }^{54}$

\section{Metabolomic NMR insights of goats and sheep serum samples}

Metabolomics serum ${ }^{1} \mathrm{H}$ NMR analyses were applied for evaluation of effects of AgNP-based cream on serum metabolome. In Fig. 5, serum ${ }^{1} \mathrm{H}$ NMR spectra are presented, which correspond to the administration of iodine solution (10\%) and AgNP-based cream.

After the exclusion of the HDO signal, for each analysed group (Fig. S4 and S5, ESI $\dagger$ ) the mean spectra were obtained. After the subtractions, between the mean spectra of iodine and AgNP-based cream for both animals' groups (Fig. S6 and S7†), some small differences in blood metabolomes are seen. Therefore, according to the ${ }^{1} \mathrm{H}$ NMR data, the two treatments did not cause differences in most NMR metabolites signals, except in the lactate signal $(\delta$ 1.33) and sugar region $(\delta 3.00-$ $4.00)$. Cited differences could be indicative for longer inflammation period in the area of the wound, which was observed during the treatment with iodine solution.

The scores in PC 1 and PC 2 showed variances of $28.2 \%$ in PC 1 and $14.8 \%$ in PC 2, and do not point to separation even after exclusion of two outliers (569_1I C and C543_8P) as illustrated in Fig. S8 (ESI $\dagger$ ). Observing the loading graph (Fig. S8 $\dagger$ ) for PC 1 and PC 2, there are no significant differences between two treatments, except the ones observed in the lactate region, confirming observation made upon analysis of the ${ }^{1} \mathrm{H}$ NMR mean spectra. Fig. $\mathrm{S} 9 \dagger(\mathrm{ESI} \dagger)$ shows the PC 1 vs. PC 2 scores, with $40.6 \%$ variance in PC 1 and $24.4 \%$ in PC 2 obtained for samples from sheep. No significant differences or trend in the separation of the samples were observed. The loadings point to lactate signal $(\delta$ 1.33) with negative value for PC 1 and positive for PC 2. Thus, prolonged inflammation in samples treated with $10 \%$ iodine solution and also the prolonged wound healing in iodine treated animals might have provoked these differences. In addition, the results of PLS-DA also did not point to a trend for separation of the groups, so it may be assumed that both treatment methods affected the metabolism of the animals in a very similar way when compared serum metabolites.

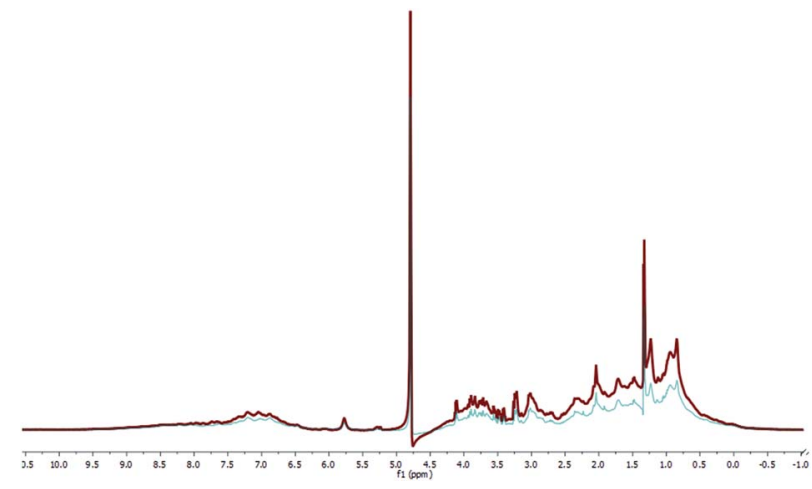

Fig. $5{ }^{1} \mathrm{H}$ NMR of serum samples taken from goats treated with iodine solution $10 \%$ (red line) and AgNP-based cream (blue line). The residual water signal (HDO of 4.20 to $5.50 \mathrm{ppm}$ ) is not shown.
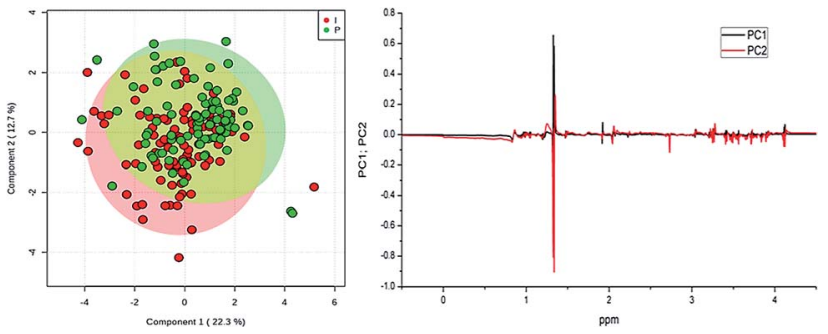

Fig. 6 Serum NMR metabolomics obtained for goats: PLS-DA scores (left, two outliers were excluded - 569_1l C and 543_8P C), and the PLS-DA loading graph (right). The green circles present goats treated with the AgNP-based cream (P) and the red circles correspond to animals treated with iodine solution $10 \%(\mathrm{I})$.

The score plot of PLS-DA method (Fig. 6) confirms that serum samples from the goats are very similar and almost superimposed (two outliers, 569_1I C and 543_8P C were excluded before performing the PLS-DA analysis).

The total cross-validation test (LOOCV) for the PLS-DA model showed $R^{2}$ inferior to 0.4 in the fifth component and $Q^{2}$ lower than 0.05 , which implies that the proposed model is valid. The distribution of the random class assignments according to the permutation test presented in the histogram for the proposed PLS-DA model showed significant results. The proposed model can be considered valid in $99 \%$ of cases since the observed statistic for 100 components is inferior to 0.01 .

By proposing the PLS-DA model, it was shown that the most important variables belong to the signals of the metabolites shown in Fig. 7. Chemical shifts on $\delta 0.85$ belong to the signal of low-density lipids, $\delta 1.25$ to the decanoic acid (capric acid, found naturally in the milk of various mammals), $\delta 1.33$ to the lactate signal $\left(3 \mathrm{H}, \mathrm{d},{ }^{3} J=7 \mathrm{~Hz}\right), \delta 2.04$ to glutamate $(\mathrm{H} 6, \mathrm{~m})$, $\delta 2.73$ to citrate $\left(2 \mathrm{H}, \mathrm{d},{ }^{3} J=16 \mathrm{~Hz}\right)$, and finally $\delta 3.26$ and 3.56 to the glucose multiple resonances. These substances showed relatively high concentration in the serum when animals were treated with the $10 \%$ iodine solution, probably as a consequence of prolonged inflammation and slower wound healing. ${ }^{55}$
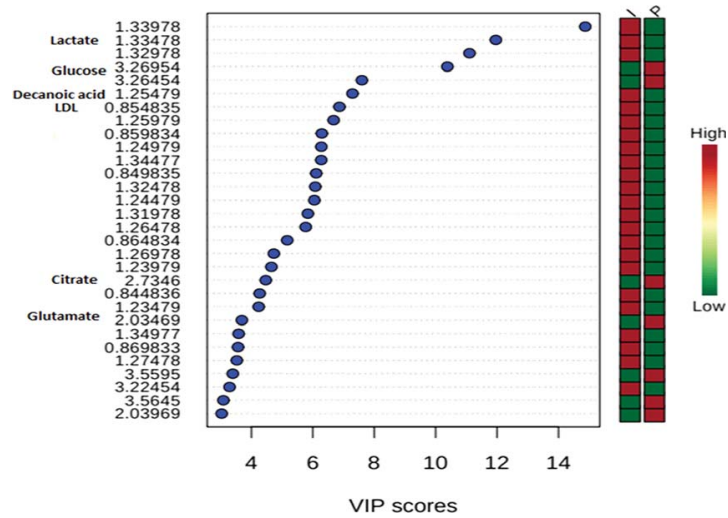

Fig. 7 Serum NMR metabolites observed for goats: importance of variables (VIP Scores) identified by PLS-DA model. The coloured boxes on the right indicate the relative concentrations of the corresponding metabolite in each group. The numbers at the left side are chemical shifts of the corresponding metabolites. Goats treated with AgNPbased cream (P) and animals treated with $10 \%$ iodine solution (I). 

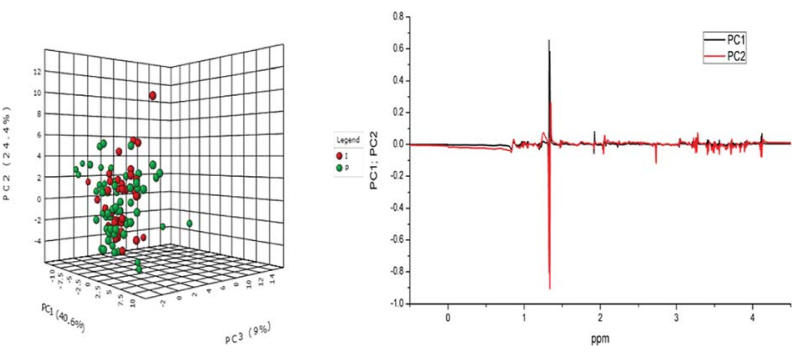

Fig. 8 Serum NMR metabolomics obtained for sheep: 3D score plot of the PLS-DA analysis (left), with excluded outliers, and the PLS-DA loading graph (right). The models were constructed from the matrix normalized by the sum and mean centred after the exclusion of outliers. The green circles present sheep treated with AgNP-based cream $(P)$ and the red circles correspond to animals treated with $10 \%$ iodine solution (I).

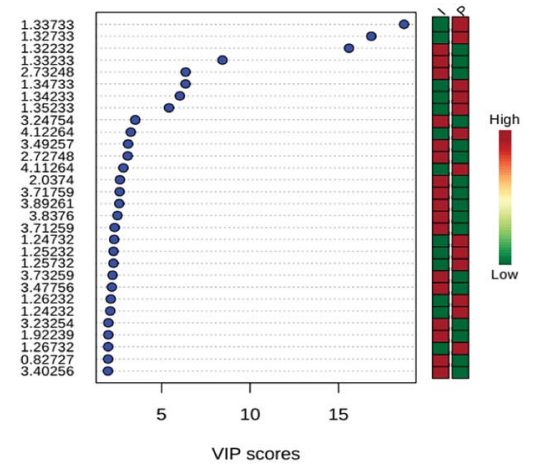

Fig. 9 Serum NMR metabolites found in sheep: importance of variables (VIP) identified by PLS-DA. The coloured boxes on the right indicate the relative concentrations of the corresponding metabolites in each studied group. Goats treated with AgNP-based cream (P) and animals treated with $10 \%$ iodine (I).

The plot graph using the PLS-DA method presented in Fig. 8, confirmed that there is no separation between NMR metabolome spectra from sheep.

The total cross-validation test (LOOCV) for the PLS-DA model showed $R^{2}$ lower than 0.4 in the fifth component and $Q^{2}$ lower than 0.05 . The model is valid in $94 \%$ of the cases. The scores of 30 metabolites' signals (Fig. 9) related to the sheep samples point to the highest importance of lactate ( $\delta 1.33$ and 4.12), for both types of treatments, of fatty acids, decanoic acid $(\delta 1.25-$ 1.26) after treatment with AgNP-based cream. Samples of sheep treated with iodine solution showed: norleucin $(\delta 3.73)$, phosphocholine $(\delta 3.27)$, citrate $(\delta 2.73)$, glutamate $(\delta 2.04)$, choline $(\delta 3.56)$ and low-density lipids $(\delta 0.85-0.86)$ as the most important VIP. Among these metabolites, decanoic acid was reported as volatile compound, ${ }^{56}$ and norleucine or aminocaproic acid also compose sheep metabolome.

\section{Inductively coupled plasma-optical emission spectrometry ICP-OES}

Concentration of $\operatorname{Ag}(\mathrm{I})$ in animal serum after the application of AgNP-based cream was determined. The highest concentration of $\mathrm{Ag}(\mathrm{I})$ was lower than $10 \mathrm{ppm}\left(\mu \mathrm{g} \mathrm{\textrm {L } ^ { - 1 }}\right)$. As seen in the histogram of silver ion concentration obtained from sheep sample 564 treated with AgNP-based cream (Fig. S10, ESI $\dagger$ ), only a slight variation of silver(I) was detected, but during treatment, the ion concentration did not exceed $10 \mathrm{ppm}$. Since the concentration of free ions in the course of treatment with commercial cream (Sulfadiazine cream 1\%) exceeds, in some cases, much more than $10 \mathrm{ppm},{ }^{57}$ we can conclude that the treatment we proposed with AgNP-based cream accumulates smaller amount of free $\mathrm{Ag}(\mathrm{I})$ ions in blood of the treated animals.

\section{Conclusions}

Cream with biogenic silver nanoparticles was prepared and used for wound treatment after surgical removal of nodules caused by caseous lymphadenitis disease in sheep and goats. With the aim to compare the proposed and conventional treatment with $10 \%$ iodine solution, serum metabolomics by NMR was done. All biochemical tests pointed to a significant clinical improvement of the AgNP-based cream treated animals without any metabolic or clinical change. These observations indicate that new treatment had no side effects on animals. In addition, the treatment with the AgNP-based cream accelerated up to 2-or 3-times wound healing. AgNP-based cream treatment improved animals' recovery from CL, as no recurrence of CL was noted in any of the treated animals.

\section{Conflicts of interest}

There are no conflicts to declare.

\section{Acknowledgements}

Authors would like to thank FAPESP, CAPES and CNPq for a scholarship (FAPESP No. 2015/12534-5), grants and financial supports (FAPESP No. 2018/06510-4 and 2014/18938-8). Also, the NMR facility at the Institute of Chemistry, University of Campinas, Campinas, SP, Brazil is kindly acknowledged.

\section{References}

1 W. M. Silva, F. A. Dorella, S. C. Soares, G. H. M. Souza, T. L. P. Castro, N. Seyffert, H. Figueiredo, A. Miyoshi, Y. L. Loir, A. Silva and V. Azevedo, A shift in the virulence potential of Corynebacterium pseudotuberculosis biovar ovis after passage in a murine host demonstrated through comparative proteomics, BMC Microbiol., 2017, 17, 55.

2 D. J. Haas, E. M. S. Dorneles, S. J. Spier, S. P. Carroll, J. Edman, V. A. Azevedo, M. B. Heinemann and A. P. Lage, Infection, genetics and evolution molecular epidemiology of Corynebacterium pseudotuberculosis isolated from horses in California, Infect., Genet. Evol., 2017, 49, 186-194.

3 W. M. Silva, E. L. Folador, S. C. Soares, G. H. M. F. Souza, A. V. Santos, C. S. Sousa, H. Figueiredo, A. Miyoshi, Y. L. Loir, A. Silva and A. Vasco, Label-free quantitative proteomics of Corynebacterium pseudotuberculosis isolates 
reveals differences between Biovars ovis and equi strains, BMC Genomics, 2017, 18, 451.

4 M. W. Paton, I. R. Rose, R. A. Hart, S. S. Sutherland, A. R. Mercy, T. M. Ellis and J. A. Dhaliwal, New infection with Corynebacterium pseudotuberculosis reduces wool production, Aust. Vet. J., 1994, 71, 47-49.

5 S. Almeida, S. Tiwari, D. Mariano, F. Souza, S. B. Jamal, N. Coimbra, R. T. Raittz, F. A. Dorella, A. F. Carvalho, F. L. Pereira, S. C. Soares, C. A. G. Leal, D. Barh, P. Ghosh, H. Figueiredo, L. F. Moura-Costa, R. W. Portela, R. Meyer, A. Silva and V. Azevedo, The genome anatomy of Corynebacterium pseudotuberculosis VD57 a highly virulent strain causing Caseous lymphad-enitis, Stand. Genomic Sci., 2016, 11, 29.

6 J. G. M. Pontes, F. B. Santana, R. W. Portela, V. Azevedo, R. J. Poppi and L. Tasic, Biomarkers of the Caseous Lymphadenitis in sheep by NMR-based metabolomics, Metabolomics, 2017, 7, 190.

7 D. M. Rhodes, K. G. Magdesian, B. A. Byrne, P. H. Kass, J. Edman and S. J. Spier, Minimum Inhibitory Concentrations of equine Corynebacterium pseudotuberculosis isolates (1996-2012), J. Vet. Intern. Med., 2015, 29, 327-332.

8 D. Abebe and T. S. Tessema, Determination of Corynebacterium pseudotuberculosis prevalence and antimicrobial susceptibility pattern of isolates from lymph nodes of sheep and goats at an organic export abattoir, Modjo, Ethiopia, Lett. Appl. Microbiol., 2015, 61, 469-476.

9 F. Bassyouni, N. Elhalwany, M. A. Rehim and M. Neyfeh, Advances and new technologies applied in controlled drug delivery system, Res. Chem. Intermed., 2015, 41, 2165-2200.

10 J. L. Clement and P. S. Jarrett, Antibacterial silver, Met.-Based Drugs, 1994, 1, 467-482.

11 M. M. Mohamed, S. A. Fouad, H. A. Elshoky, G. M. Mohammed and T. A. Salaheldin, Antibacterial effect of gold nanoparticles against Corynebacterium pseudotuberculosis, Int. J. Vet. Sci. Med., 2017, 5, 23-29.

12 M. da C. A. Sá, J. L. A. Veschi, G. B. Santos, E. S. Amanso, S. A. S. Oliveira, R. A. Mota, G. Veneroni-Gouveia and M. M. Costa, Activity of disinfectants and biofilm production of Corynebacterium pseudotuberculosis, Pesqui. Vet. Bras., 2013, 33(11), 1319-1324.

13 I. Chopra, The increasing use of silver-based products as antimicrobial agents: a useful development or a cause for concern?, J. Antimicrob. Chemother., 2007, 59, 587-590.

14 M. Yamanaka, K. Hara and J. Kudo, Bactericidal actions of a silver ion solution on Escherichia coli, studied by energyfiltering transmission electron microscopy and proteomic analysis, Appl. Environ. Microbiol., 2005, 71, 7589-7593.

15 M. Vogler, D. Dinsdale, M. J. S. Dyer and G. M. Cohen, Bcl-2 inhibitors: small molecules with a big impact on cancer therapy, Cell Death Differ., 2009, 16, 360-367.

16 W. K. Jung, H. C. Koo, K. W. Kim, S. Shin, S. H. Kim and Y. H. Park, Antibacterial activity and mechanism of action of the silver ion in Staphylococcus aureus and Escherichia coli, Appl. Environ. Microbiol., 2008, 74, 2171-2178.
17 D. Ballottin, S. Fulaz, F. Cabrini, J. Tsukamoto, N. Durán, O. L. Alves and L. Tasic, Antimicrobial textiles: Biogenic silver nanoparticles against Candida and Xanthomonas, Mater. Sci. Eng., C, 2017, 75, 582-589.

18 V. L. Praba, M. Kathirvel, K. Vallayyachari, K. Suren-dar, M. Muthuraj, P. J. Jesuraj, S. Govindarajan and K. V. Raman, Bactericidal effect of silver nanopar-ticles against Mycobacterium tuberculosis, J. Bionanosci., 2013, 7, 282-287.

19 R. Salomoni, P. Léo, A. F. Montemor, B. G. Rinaldi and M. / F. A. Rodrigues, Antibacterial effect of silver nanoparticles in Pseudomonas aeruginosa, Nanotechnol., Sci. Appl., 2017, 10, 115-121.

20 N. Durán, G. Nakazato and A. B. Seabra, Antimicrobial activity of biogenic silver nanoparticles, and silver chloride nanoparticles: an overview and comments, Appl. Microbiol. Biotechnol., 2016, 100, 6555-6570.

21 N. Durán, M. Durán, M. B. Jesus, A. B. Seabra, W. J. Fávaro and G. Nakazato, Silver nanoparticles: A new view on mechanistic aspects on antimicrobial activity, Nanomedicine, 2016, 12, 789-799.

22 L. Tasic, R. W. D. Portela, C. H. N. Barros, J. G. M. Pontes, D. Stanisic, and N. Durán, Formulação Farmacêutica e seu uso, Patente de Invenção, BR 102017014836 0, 2017.

23 J. Tian, K. K. Y. Wong, C. Ho, C. N. Lok, W. Y. Yu, C. M. Che, J. F. Chiu and P. K. Tam, Topical delivery of silver nanoparticles promotes wound healing, ChemMedChem, 2007, 2, 129-136.

24 J. E. Barrett, Handbook of Experimental Pharmacology, Springer Nature, 1978, vol. 49, ISSN: 0171-2004.

25 C. Rigo, L. Ferroni, I. Tocco, M. Roman, I. Munivra-na, - Gardin, W. R. L. Cairns, V. Vindigni, B. Azzena, C. Barbante and B. Zavan, Active silver nano-particles for wound healing, Int. J. Mol. Sci., 2013, 14, 4817-4840.

26 M. B. Dreifke, A. A. Jayasuriya and A. C. Jayasuriya, Current wound healing procedures and potential care, Mater. Sci. Eng., C, 2015, 48, 651-662.

27 J. Jain, S. Arora, J. M. Rajwade, P. Omray, S. Khandelwal and K. M. Paknikar, Silver nanoparticles in therapeutics: Development of an antimicrobial gel formulation for topical use, Mol. Pharm., 2009, 6, 1388-1401.

28 S. Halouska, B. Zhang, R. Gaupp, S. Lei, E. Snell, R. J. Fenton, R. G. Barletta, G. A. Somerville and R. Powers, Revisiting protocols for the NMR analysis of bacterial metabolomes, Journal of Integrated OMICS, 2013, 3, 120-137.

29 A. C. Dona, M. Kyriakides, F. Scott, E. A. Shephard, D. Varshavi, K. Veselkov and J. R. Everett, A guide to the identification of metabolites in NMR-based metabonomics/ metabolomics experiments, Comput. Struct. Biotechnol. J., 2016, 14, 135-153.

30 M. M. W. B. Hendriks, F. A. van Eeuwijk, R. H. Jellema, J. A. Westerhuis, T. H. Reijmers, H. C. J. Hoefsloot and A. K. Smilde, Data-processing strategies for metabolomics studies, Trends Anal. Chem., 2011, 30, 1685-1698.

31 J. G. M. Pontes, A. J. M. Brasil, G. C. F. Cruz, R. N. Souza and L. Tasic, NMR-based metabolomics strategies: plants, animals and humans, Anal. Methods, 2017, 9, 1078-1096. 
32 R. Bro and A. K. Smilde, Principal component analysis, Anal. Methods, 2014, 6, 2812-2831.

33 J. Xia and D. S. Wishart, Metabolomic data processing, analysis, and interpretation using MetaboAnalyst, Curr. Protoc. Bioinf., 2011, 034, 14.10.1-14.10.48.

$34 \mathrm{~J}$. Xia and D. S. Wishart, Using MetaboAnalyst 3.0 for comprehensive metabolomics data analysis, Curr. Protoc. Bioinf., 2016, 55, 14.10.1-14.10.91.

35 A. Erban, B. De and C. Wagner, US Pat., 10054066, 2013, vol. $2,12$.

36 P. S. Gromski, H. Muhamadali, D. I. Ellis, Y. Xu, E. Correa, M. L. Turner and R. Goodacre, A tutorial review: Metabolomics and partial least squares-discriminant analysis - a marriage of convenience or a shotgun wedding, Anal. Chim. Acta, 2015, 879, 10-23.

37 E. Szymanska, E. Saccenti, A. K. Smilde and J. A. Westerhuis, Double-check: validation of di-agnostic statistics for PLS-DA models in metabolomics studies, Metabolomics, 2012, 8, 316.

38 C. H. N. Barros, G. C. F. Cruz, W. Mayrink and L. Tasic, Biobased synthesis of silver nanoparticles from orange waste: effects of distinct biomolecule coatings on size, morphology, and antimicrobial activity, Nanotechnol., Sci. Appl., 2018, 11, 1-14.

39 L. B. Santiago, R. R. Pinheiro, A. S. Rodrigues, L. Chapaval, I. F. Brito and F. G. C. Sousa, Avaliação in vitro da sensibilidade da Corynebacterium pseudotuberculosis frente a diferentes tipos de antissépticos e desinfetantes e determinação de sua curva de crescimento, Arq. Inst. Biol., 2010, 77, 593-600.

40 N. Durán, P. D. Marcato, R. De Conti, O. L. Alves, F. T. M. Costa and M. Brocchi, Potential use of silver nanoparticles on pathogenic bacteria, their toxicity and possible mechanisms of action, J. Braz. Chem. Soc., 2010, 21, 949-959.

41 K. K. Y. Wong and X. Liu, Silver nanoparticles - the real "silver bullet " in clinical medicine?, MedChemComm, 2010, 1, 125-131.

42 C. Marambio-Jones and E. M. V. Hoek, A review of the antibacterial effects of silver nanomaterials and potential implications for human health and the environment, $J$. Nanopart. Res., 2010, 12, 1531-1551.

43 C. Brambilla, A. Sánchez-Chardi, M. Pérez-Trujillo, E. Julián and M. Luquin, Cyclopropanation of $\alpha$-mycolic acids is not required for cording in Mycobacterium brumae and Mycobacterium fallax, Microbiology, 2012, 158, 1615-1621.

44 H. Marrakchi, M. A. Lanéelle and M. Daffé, Review mycolic acids: Structures, biosynthesis, and beyond, Cell Press, 2014, vol. 21, pp. 67-85.

45 J. C. Ruiz, V. D'Afonseca, A. Silva, A. Ali, A. C. Pinto, A. R. Santos, et al., Evidence for reductive genome evolution and lateral acquisition of virulence functions in two Corynebacterium pseudotuberculosis strains, PLoS One, 2011, 6, e18551.
46 V. Thammavongsa, J. W. Kern, D. M. Missiakas and O. Schneewind, Staphylococcus aureus synthesizes adenosine to escape host immune responses, J. Exp. Med., 2009, 206, 2417-2427.

47 Y. Yuan, Q. Peng and S. Gurunathan, Effects of silver nanoparticles on multiple drug-resistant strains of Staphylococcus aureus and Pseudomonas aeruginosa from mastitis-infected goats: An alternative approach for antimicrobial therapy, Int. J. Mol. Sci., 2017, 18, 569.

48 I. Sondi and B. Salopek-Sondi, Silver nano-particles as antimicrobial agent: a case study on E. coli as a model for Gram-negative bacteria, J. Colloid Interface Sci., 2004, 275, 177-182.

49 N. Beyth, I. Yudovin-Farber, M. Perez-Davidi, A. J. Domb and E. I. Weiss, Polyethyleneimine nanoparticles incorporated into resin composite cause cell death and trigger biofilm stress in vivo, Proc. Natl. Acad. Sci. U. S. A., 2010, 107, 22038-22043.

50 B. S. Series. Edition S., Dry Skin and Moisturizers, 2006.

51 J. Cabral, A. E. Ryan, M. D. Griffin and T. Ritter, Extracellular vesicles as modulators of wound healing, Adv. Drug Delivery Rev., 2018, 129, 394-406.

52 M. D. Leonida and I. Kumar, Bionanomaterials for skin regeneration, Springer International Publishing, 1st edn, 2016, p. 144.

53 J. Stojkovska, Z. D. I. Jancic, B. Bufan, M. M. R. Jankovic, V. Miskovic-Stankovic and B. Obradovic, Comparative in vivo evaluation of novel formulations based on alginate and silver nanoparticles for wound treatments, J. Biomater. Appl., 2018, 32(9), 1197-1211.

54 M. M. P. Silva, M. I. F. de Aguiar, A. B. Rodrigues, M. C. Miranda, M. A. M. Araújo, I. L. T. P. Rolim and A. A. A. Souza, The use of nanoparticles in wound treatment: a systematic review, Rev. Esc. Enferm. USP, 2017, 51, e03272.

55 R. Haas, J. Smith, V. Rocherros, S. Nadkarni, T. MonteroMelendez, F. D'Acquisto, E. J. Bland, M. Bombardieri, C. Pitzalis, M. Perretti, F. M. Marelli-Berg and C. Mauro, Lactate regulates metabolic and pro- inflammatory circuits in control of $\mathrm{T}$ cell migration and effector functions, PLOS Biol., 2015, 13, e1002202.

56 V. T. de Souza, É. P. D. de Franco, M. E. de Araújo, M. C. Messias, F. B. Priviero, A. C. Frankland Sawaya and P. Oliveira Carvalho, Characterization of the antioxidant activity of aglycone and glycosylated derivatives of hesperetin: an in vitro and in vivo study, J. Mol. Recognit., 2015, 29, 80-87.

57 G. M. Davolli, K. N. Beavers, V. Medina, J. L. Sones, C. R. F. Pinto, D. L. Paccamonti and R. C. Causey, Concentrations of Sulfadiazine and Trimethoprim in Blood and Endometrium of Mares After Administration of an Oral Suspension, J. Equine Vet. Sci., 2018, $1 \mathrm{e} 4$. 